\title{
Genome Sequence Resource and Annotation of Calonectria montana, an Economically Important Pathogen with a Broad Host Range
}

\author{
Yuee Tian, ${ }^{\dagger}$ Yan Li, Shuhe Wang, Jiaxuan He, Di Sun, Shengming Liu, Genqiang Chen, and \\ Zhiping $\mathrm{Che}^{\dagger}$
}

Department of Plant Protection, Henan University of Science and Technology, Luoyang, 471000, Henan Province, P. R. China

\section{Genome Announcement}

Calonectria montana is an important soilborne fungal pathogen with a broad host range which causes serious diseases on forestry, agricultural, and horticultural plants. Here, we report a complete genome assembly of C. montana PCam007 (assembly $=59.3 \mathrm{Mb}$ and contig $\mathrm{N}_{50}$ : $6.36 \mathrm{Mb}$ ), using a combination of Illumina paired-end and Oxford Nanopore Technologies (ONT) long-read sequencing data. In total, 14,771 protein-coding genes and 477 noncoding RNAs were identified, among which 14,365 genes were annotated. Benchmarking universal single-copy orthology (BUSCO) analysis indicated that $98.50 \%$ of the BUSCOs were complete whereas $98.20,0.40$, and $0.10 \%$ were single copy, duplicated, and fragmented, respectively. The availability of this high-quality genome resource is expected to facilitate research on the evolution, host adaption, and pathogenicity of $C$. montana and to provide new insight into the infection mechanisms of $C$. montana and disease management.

Calonectria represents a genus of phytopathogenic ascomycetous fungi with a worldwide distribution. Species of this genus have a broad host range that includes more than 335 plant species, distributed across nearly 100 plant families (Wu and Chen 2021). Calonectria spp. cause important diseases on various forestry, horticultural, and agricultural crops (Crous 2002; Liu et al. 2020). Symptoms associated with infection by these fungi include stem cankers and leaf and shoot blight, as well as root rot.

The significance of Calonectria spp. has justified many taxonomic studies on these fungi in recent years. Thus far, 169 species have been identified based on a combination of DNA sequence data and morphological characteristics (Wu and Chen 2021). China has relatively high Calonectria spp. diversity. Up to now, 23 species of Calonectria have been described (Wu and Chen 2021). Of these, most species were isolated from Eucalyptus plants or soils. C. montana is a newly described species which was first isolated from natural forest soil in central China in 2016 (Liu and Chen 2017). It was later described in Poland to cause seedling damping-off on Pinus sylvestris and Picea abies (Stepniewska et al. 2020). A recent study reported that the species can cause root rot in sugar beet (Shao and Li 2021). Additionally, C. montana can also cause brown spot disease on tree peony, a well-known Chinese traditional flower widely cultivated all over the world (Fig. 1A) (Cheng 2007) (unpublished data).

\footnotetext{
${ }^{\dagger}$ Corresponding authors: Y. E. Tian; yueetian@ haust.edu.cn, and Z. E. Che; zhipingche@163.com
}

The author(s) declare no conflict of interest.

Accepted for publication 27 February 2022.

\section{Funding}

Support was provided by the National Natural Science Foundation of China grant 31901863 .

\section{Keywords}

Calonectria montana, brown spot disease, fungus-plant interactions, genomics, metabolomics, Oxford Nanopore Technology, peony (Paeonia Suffruticosa), proteomics, whole-genome sequencing 


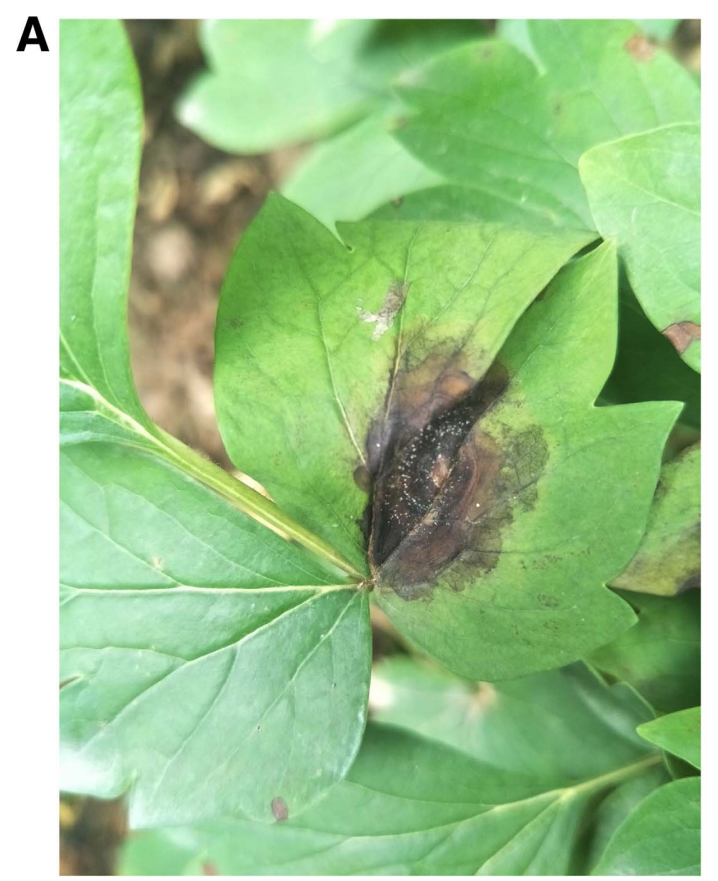

B

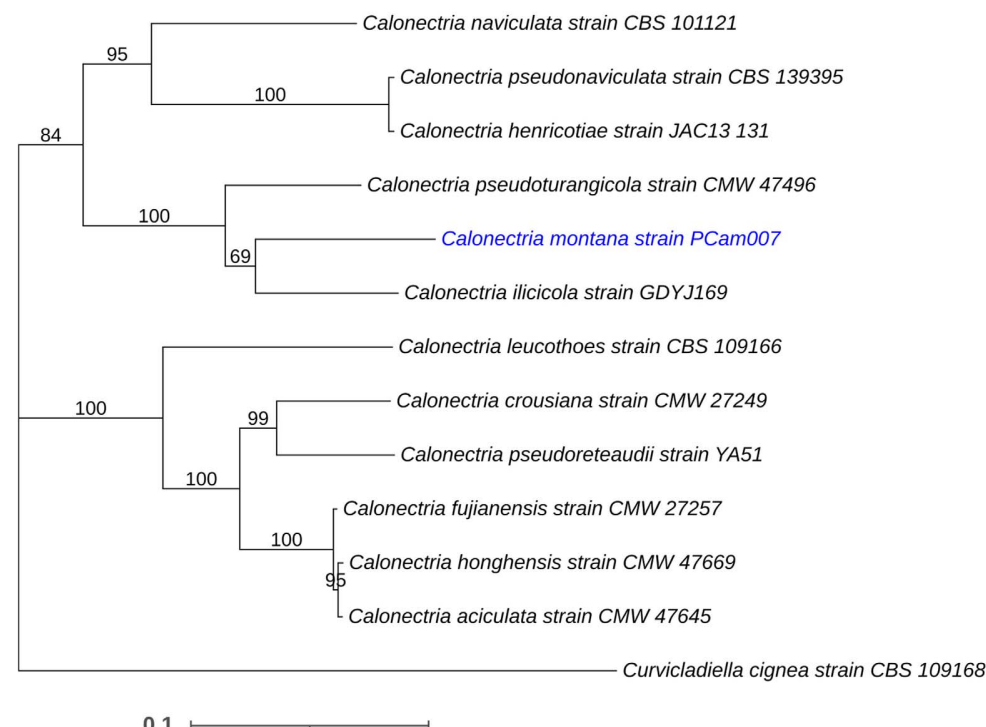

0.1

Fig. 1. A, Disease phenotype of Calonectria montana on leave of tree peony and B, phylogenetic tree of the genus Calonectria based on maximum likelihood (ML) analyses of combined DNA dataset of act, cmdA, his3, ITS, LSU, rpb2, tef1, tub2, MAT1-1-1, and MAT1-2-1 gene sequences. Sequences of the other Calonectria spp. were downloaded from NCBI. Bootstrap values for maximum-likelihood analyses are presented at the branches.

The isolate PCam007 in this study was first isolated from infected tree peony leaves collected from Sui and Tang Dynasty Town Botanic Gardens, located in Luoyang City, China. Based on morphological characteristics and multigene phylogenetic analyses of the internal transcribed spacer (Liu et al. 2020), PCam007 was identified as C. montana. A pathogenicity test on 15 tree peony varieties showed that the pathogen is highly virulent toward all tested varieties, even under no-wounding inoculation (unpublished data). Whole-genome sequencing of the C. montana isolate PCam007 was conducted by ONT long-read sequencing (for de novo assembly) and Illumina short-read sequencing (for polishing). The overall aim was to facilitate future research regarding this important fungus, especially relating to its taxonomy, population genetics, and pathogenicity.

High-quality genomic DNA of PCam007 was extracted and purified from 3-day-old fresh mycelium using the cetyltrimethylammonium bromide method, and the DNA quality and concentration were determined by $0.75 \%$ agarose gel electrophoresis on a NanoDrop One spectrophotometer (Thermo Fisher Scientific) and a Qubit v3.0 Fluorometer (Life Technologies, Carlsbad, CA, U.S.A.) respectively. It was then randomly sheared by a Covaris ultrasonic disruptor. Illumina sequencing pair-end libraries with insert size of $300 \mathrm{bp}$ were prepared using a Nextera DNA Flex Library Prep Kit (Illumina, San Diego, CA, U.S.A.). Sequencing was performed using the Illumina NovaSeq platform (Illumina). Raw reads were cleaned to discard low-quality reads (reads with adaptors or unknown nucleotides or reads with more than $20 \%$ low-quality bases) using the SOAPnuke (v2.1.4) tool and, after data filtering, clean data were used for subsequent analyses.

For ONT sequencing, the libraries were prepared using the SQK-LSK109 ligation kit and the standard protocol. The purified library was loaded onto primed R9.4 Spot-On Flow Cells and sequenced using a PromethION sequencer (Oxford Nanopore Technologies, Oxford, U.K.) with 48-h runs. Base-calling analyses of raw data were performed using the ONT GUPPY software (v0.3.0).

We obtained $12.78 \mathrm{~Gb}$ of ONT reads, with $\mathrm{N}_{50}$ length of $20.79 \mathrm{~kb}$, and $4.64 \mathrm{~Gb}$ of paired-end Illumina reads (Table 1). The sequencing depth of ONT reads and Illumina reads represent $215 \times$ and $81 \times$ genome coverage, respectively (Table 1). To obtain genome assembly, we exploited NextDenovo v2.3.1 to generate a draft assembly, using pure ONT reads, and NextPolish v1.3.1 to polish the assembly using Illumina reads. The final genome 
Table 1. Genome features of Calonectria montana PCam007

\begin{tabular}{|c|c|}
\hline Feature & Isolate PCam007 \\
\hline Oxford Nanopore Technologies (ONT) long reads $(\mathrm{Gb})^{\mathrm{a}}$ & 12.78 (approximately $215 \times$ ) \\
\hline $\mathrm{N}_{50}$ of ONT reads $(\mathrm{kb})$ & 20.79 \\
\hline Mean ONT read length $(\mathrm{kb})$ & 11.88 \\
\hline Maximum ONT read length $(\mathrm{kb})$ & 195.00 \\
\hline Illumina short reads $(\mathrm{Gb})^{\mathrm{a}}$ & 4.64 (approximately $81 \times$ ) \\
\hline Estimated genome size (Mb) & 57.16 \\
\hline Estimated genome repeats $(\mathrm{Mb})$ & 11.04 \\
\hline Estimated genome heterozygosity (\%) & 0.096 \\
\hline Assembly size $(\mathrm{Mb})$ & 59.30 \\
\hline Contig number & 13 \\
\hline Contig $\mathrm{N}_{50}(\mathrm{Mb})$ & 6.36 \\
\hline Contig $\mathrm{N}_{90}(\mathrm{Mb})$ & 14.00 \\
\hline Average contig length $(\mathrm{Mb})$ & 4.56 \\
\hline Maximum contig length $(\mathrm{Mb})$ & 9.32 \\
\hline GC content (\%) & 47.25 \\
\hline Illumina reads mapping rate & 97.17 \\
\hline Benchmarking universal single-copy orthology (BUSCO) complete (\%) & 98.50 \\
\hline Fragmented BUSCOs (\%) & 0.10 \\
\hline Missing BUSCOs (\%) & 1.30 \\
\hline Noncoding RNA & 477 \\
\hline Protein-coding genes ${ }^{\mathrm{b}}$ & 14,771 \\
\hline Genes annotated by Pfam & 12,056 \\
\hline Genes annotated by Gene Ontology & 8,734 \\
\hline Genes annotated by Kyoto Encyclopedia of Genes and Genomes & 4,868 \\
\hline Genes annotated by Carbohydrate Active Enzymes & 539 \\
\hline Genes annotated by Clusters of Orthologous Groups & 1,380 \\
\hline Genes annotated by the Transporter Classification Database & 2,442 \\
\hline Cytochrome P450s & 1,721 \\
\hline Transmembrane proteins & 3,058 \\
\hline Secreted proteins & 1,555 \\
\hline
\end{tabular}

a Genome heterozygosity, size, and repeat content were estimated by kmer-based method GenomeScope (version 1.0.0) using Illumina short reads.

${ }^{\mathrm{b}}$ Predictions were based on a previously described pipeline (Liang et al. 2018).

assembly was $59.30 \mathrm{Mb}$ in length and contained 13 scaffolds (Table 1). The assembly size is within the range of published Calonectria genomes (C. aciculata, $C$. crousiana, C. fujianensis, C. henricotiae, C. honghensis, C. leucothoes, C. naviculata, C. pseudonaviculata, C. pseudoreteaudii, C. pseudoturangicola, and C. ilicicola), ranging from 47.50 to $68.97 \mathrm{Mb}$. The phylogenetic tree, based on maximum-likelihood analyses of a 10-gene (act, cmdA, his3, ITS, LSU, rpb2, tef1, tub2, MAT1-1-1, and MAT1-2-1) combined DNA dataset, showed that the C. montana isolate PCam007 constitute a single branch (Fig. 1B) (Liu et al. 2019; Liu et al. 2020; Zhang et al. 2020). The $\mathrm{N}_{50}$ scaffold length and the maximum scaffold length were 4.56 and $9.32 \mathrm{Mb}$, respectively. The assembly GC content was $47.25 \%$, which is close to the reported Calonectria genomes (Liu et al. 2019; Liu et al. 2020). The completeness of PCam007 genome assembly was evaluated through benchmarking universal single-copy orthologs (BUSCO) v4.1.4 with 'eukaryota_odb10' $(n=255)$ as reference dataset (Seppey et al. 2019). This analysis indicates that genome assembly of isolate PCam007 contains $98.50 \%$ completed BUSCOs, $0.10 \%$ fragmented BUSCOs, and $1.30 \%$ missing BUSCOs (Table 1).

Protein-encoding gene prediction was performed by BRAKER2 (Brůna et al. 2021). In total, 14,771 protein-encoding genes were predicted (Table 1). To gain a functional gene annotation, we annotated whole-genome protein-encoding genes with Pfam, Gene Ontology (GO), Kyoto Encyclopedia of Genes and Genomes (KEGG), Clusters of Orthologous Groups (COG), and carbohydrate active enzymes (CAZymes) databases (Guo et al. 2021). We identified a total of 12,056 proteins with Pfam domains, 8,734 genes with GO items, 4,868 genes involved in different KEGG pathways, and 1,380 COG genes. In total, 759 genes were mapped to the CAZyme database and encoded carbohydrate-active enzymes and 3,058 genes encoded transmembrane proteins as performed by Tmhmm v5.0. INFERNAL v1.1.2 analysis predicted 477 noncoding RNAs, including 341 transfer RNAs, 89 ribosomal RNAs, 43 small nuclear RNAs, and four small RNA. 


\section{Data Availability}

The availability of this high-quality genome resource will accelerate studies with $C$. montana regarding comparative genomics, evolution, and population genetics. This advanced understanding on $C$. montana will further improve strategies for disease management. The assembled genome has been deposited at GenBank under the accession number JAIZZI000000000.

\section{Author-Recommended Internet Resource}

SOAPnuke (v2.1.4) tool: https://github.com/BGl-flexlab/SOAPnuke

\section{Literature Cited}

Brůna, T., Hoff, K. J., Lomsadze, A., Stanke, M., and Borodovsky, M. 2021. BRAKER2: Automatic eukaryotic genome annotation with GeneMark-EP+ and AUGUSTUS supported by a protein database. NAR Genomics Bioinf. 3: lqaa108.

Cheng, F. Y. 2007. Advances in the breeding of tree peonies and a cultivar system for the cultivar group. Int. J. Plant Breed. 1:89-104.

Crous, P. W. 2002. Taxonomy and Pathology of Cylindrocladium (Calonectria) and Allied Genera. American Phytopathological Society, St. Paul, MN, U.S.A.

Guo, H. Y., Bao, J. D., Lin, L. Y., Wang, Z. X., Shi, M. Y., Huang, Y. T., Wang, R. B., Li, B. J., Liu, P. Q., and Chen, Q. H. 2021. Genome sequence data of Peronophythora litchi, an oomycete pathogen causing litchi downy blight. Mol. Plant-Microbe Interact. 34:707-710.

Liang, X., Wang, B., Dong, Q., Li, L., Rollins, J. A., Zhang, R., and Sun, G. 2018. Pathogenic adaptations of Colletotrichum fungi revealed by genome wide gene family evolutionary analyses. PLoS One 13:e0196303.

Liu, F., Chen, S., Ferreira, M. A., Chang, R., Sayari, M., Kanzi, A. M., Wingfield, B. D., Wingfield, M. J., Pizarro, D., Crespo, A., Divakar, P. K., de Beer, Z. W., and Duong, T. A. 2019. Draft genome sequences of five Calonectria species from Eucalyptus plantations in China, Celoporthe dispersa, Sporothrix phasma and Alectoria sarmentosa. IMA Fungus 10:22.
Liu, Q. L., and Chen, S. F. 2017. Two novel species of Calonectria isolated from soil in a natural forest in China. MycoKeys 26:25-60.

Liu, Q. L., Li, J. Q., Wingfield, M. J., Duong, T. A., Wingfield, B. D., Crous, P. W., and Chen, S. F. 2020. Reconsideration of species boundaries and proposed DNA barcodes for Calonectria. Stud. Mycol. 97:100106.

Seppey, M., Manni, M., and Zdobnov, E. M. 2019. BUSCO: Assessing genome assembly and annotation completeness. Methods Mol. Biol. 1962:227-245. 31020564.

Shao, H. T., and Li, H. 2021. First report of root rot caused by Calonectria montana on Sugar beet in Heilongjiang province, China. Plant Dis. 105: 3292.

Stẹpniewska, H., Jankowiak, R., Boroń, P., and Woźniak, K. 2020. First report of Calonectria montana causing damping-off disease on pine and spruce seedlings in Europe. For. Pathol. 50:e12595.

Wu, W., and Chen, S. 2021. Species diversity, mating strategy and pathogenicity of Calonectria species from diseased leaves and soils in the Eucalyptus plantation in Southern China. J. Fungi (Basel) 7:73.

Zhang, D., Gao, F., Jakovlić, I., Zou, H., Zhang, J., Li, W. X., and Wang, G. T. 2020. PhyloSuite: An integrated and scalable desktop platform for streamlined molecular sequence data management and evolutionary phylogenetics studies. Mol. Ecol. Resour. 20:348-355. 